\title{
Hydrochemistry and Stable Isotope Indication of Mineral Water and Surface Water in Changbai Mountain, China
}

\author{
Yihan $\mathrm{Li}^{1}$, jianmin Bian ${ }^{2}$, Jialin $\mathrm{Li}^{1}$, Yuxi $\mathrm{Ma}^{1}$, and Jesus Anguiano ${ }^{3}$ \\ ${ }^{1}$ Jilin University \\ ${ }^{2}$ JiLin University \\ ${ }^{3}$ University of Guanajuato
}

January 25, 2021

\begin{abstract}
Changbai Mountain is the source region of Songhua River, Tumen River and Yalu River. It is a famous concentrated distribution area of high quality mineral water in China, which has great economic value. Antu County is one of the main distribution areas of basalt and mineral water in Changbai Mountain. The distribution of mineral springs has a strong hydraulic relationship with surface water, which constitutes abundant recharge reserves. It is necessary to study the hydrochemical characteristics and relationship between surface water and mineral water, so as to provide a theoretical basis for further discussion on the formation process and rational utilization of mineral water resources in Changbai Mountain. 57 water samples from 2016 to 2020 were collected, including precipitation, mineral water and surface water. Geostatistics, Piper and Gibbs diagram are used to analyze the hydrochemical characteristics. Hydrochemical component tracing and stable environment isotope technology with end-number calculation are used to reveal the transformation relationship between mineral water and surface water. Results demonstrate: Surface water and mineral water are weak alkaline and low salinity. The hydrochemical type of mineral water is mainly $\mathrm{HCO} 3-\mathrm{Na} \cdot \mathrm{Mg} \cdot \mathrm{Ca}$ and of surface water is mainly $\mathrm{HCO} 3-\mathrm{Na}$. The isotopes of precipitation are more enriched in summer due to the influence of water vapor source and temperature effect. The temporal and spatial differences between $\mathrm{D}$ and $18 \mathrm{O}$ of mineral water is obvious, and isotopes are enriched abundant in autumn. The recharge source and interaction between mineral water and surface water have changes in different periods. In summer, mineral springs in Erdaobai River and Sandaobai River watershed replenished surface water, and the recharge proportion is more than $60 \%$. The interaction between Toudao Baihe river and mineral water is weak. In autumn, precipitation is the main recharge for mineral water and surface water.
\end{abstract}

\section{Hosted file}

Hydrochemistry and Stable Isotope Indication of Mineral Water and Surface Water in Changbai Mountain, available at https://authorea.com/users/391554/articles/505623-hydrochemistry-and-stableisotope-indication-of-mineral-water-and-surface-water-in-changbai-mountain-china 

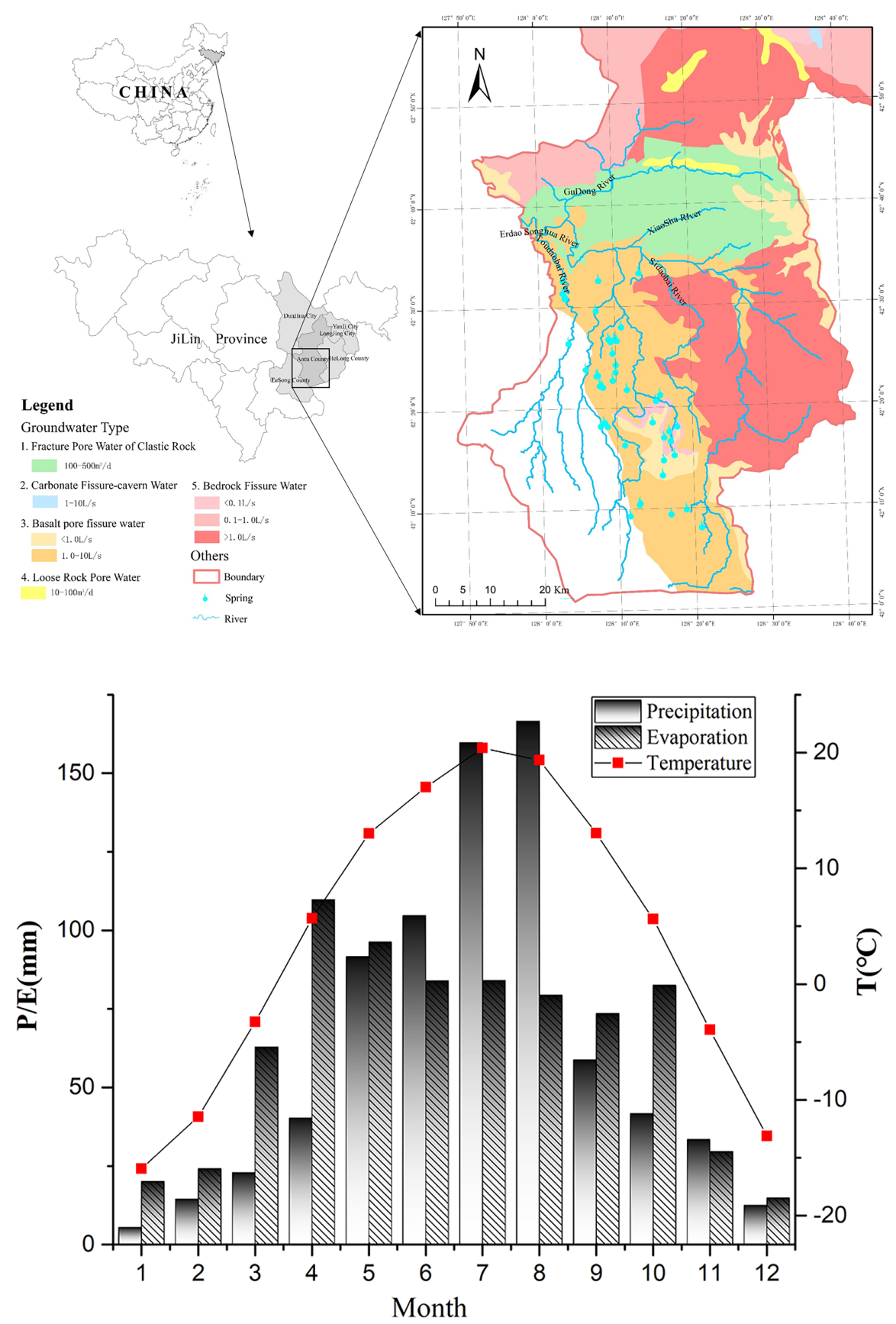


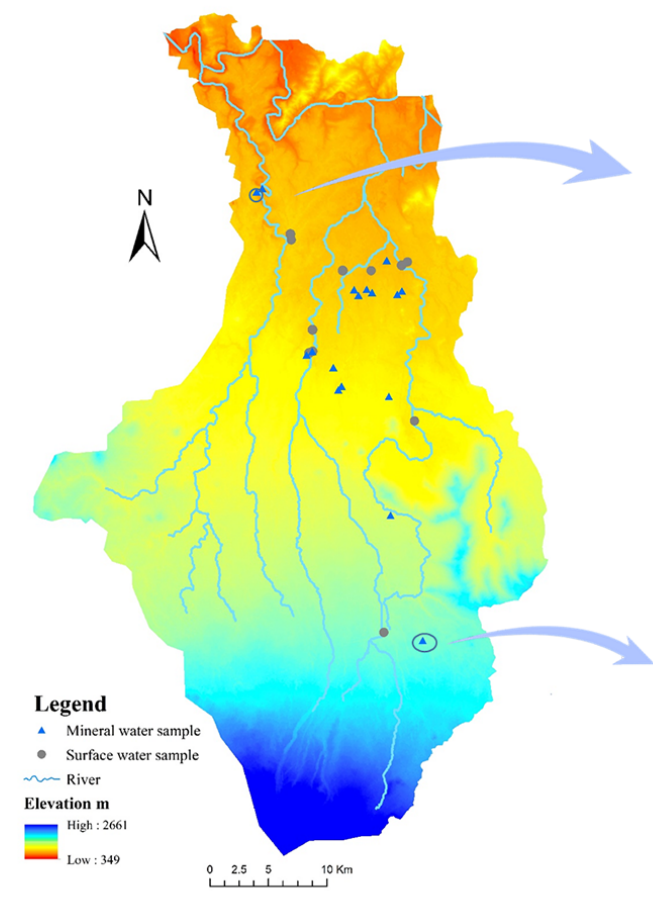

(a) The spatial distribution of water samples

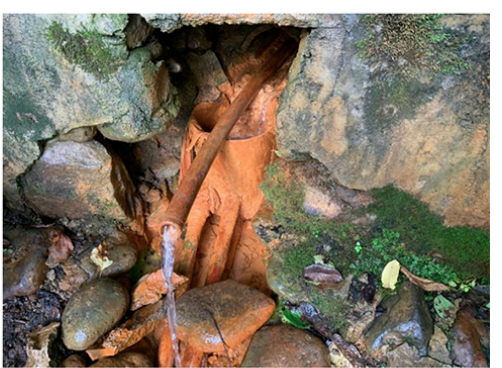

(b) Yaoshui Spring

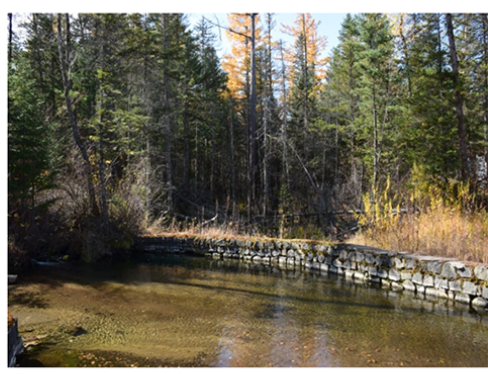

(c) Baishan Spring overflows into river
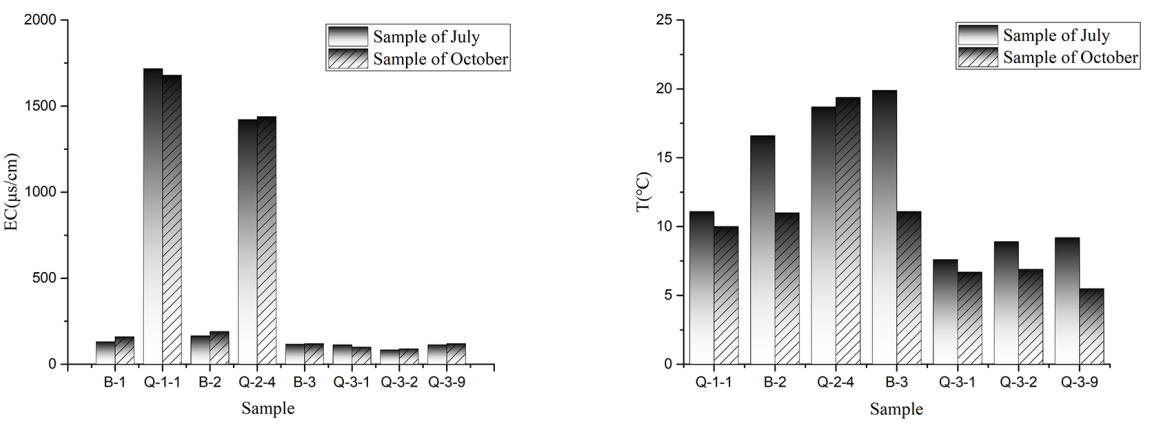

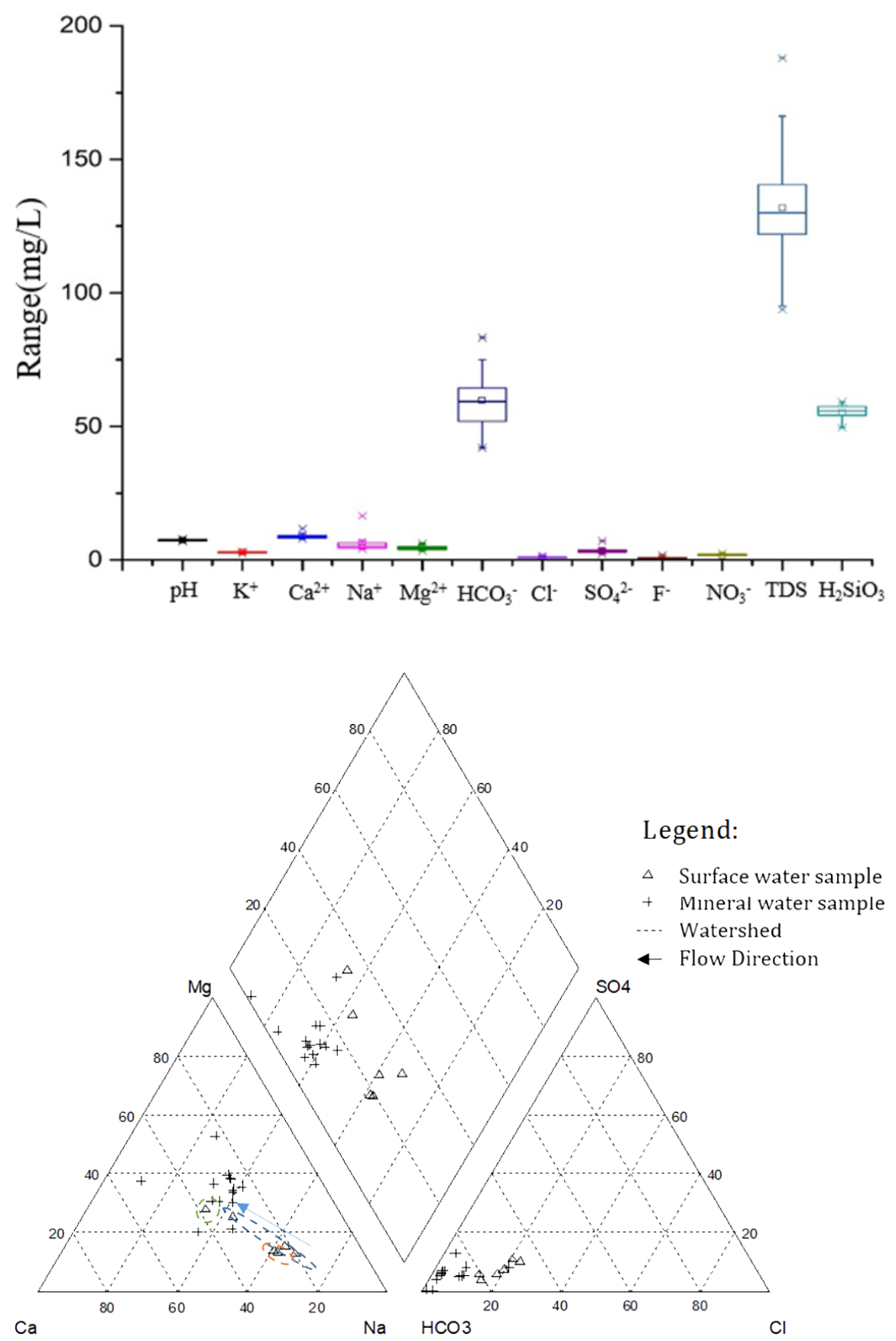

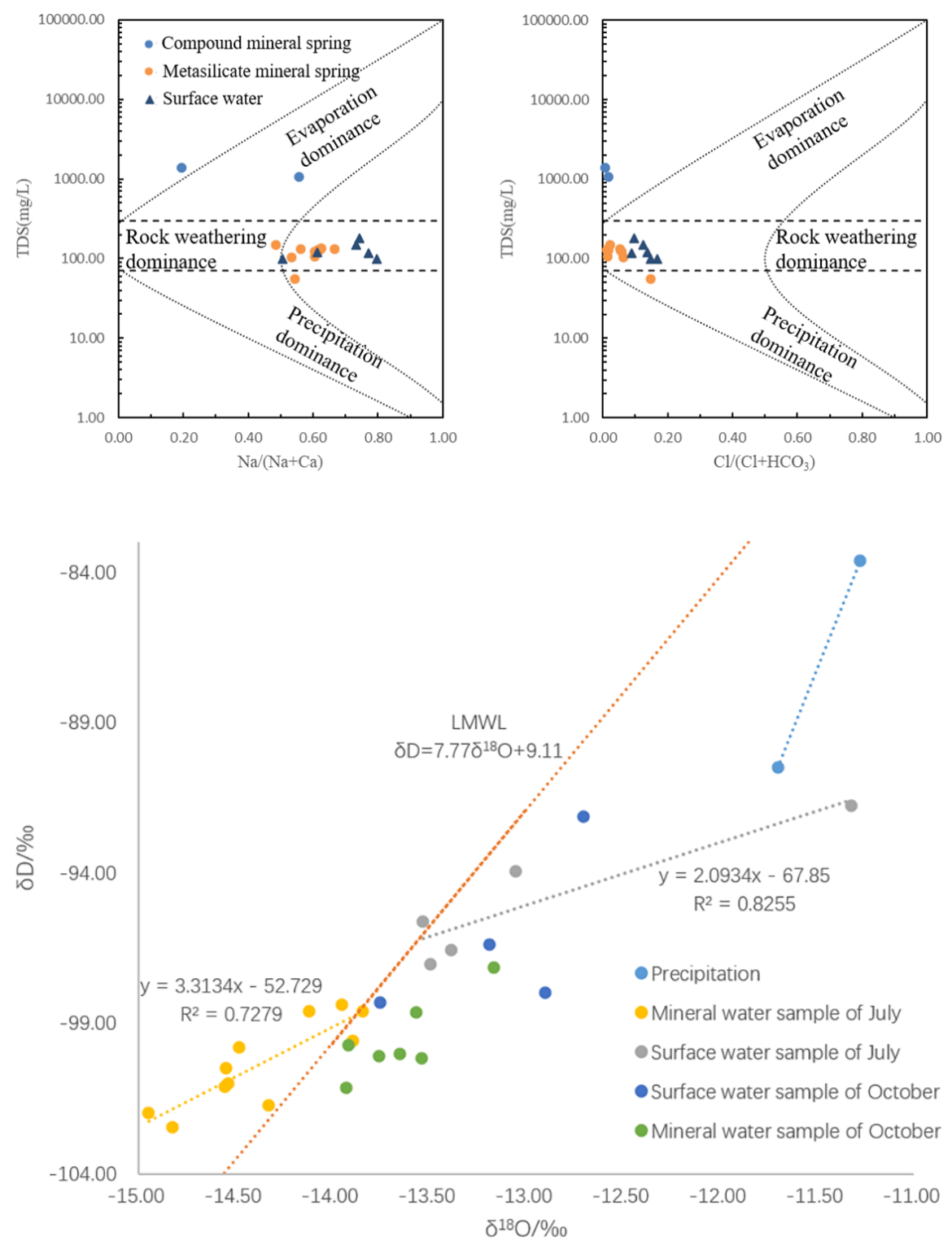

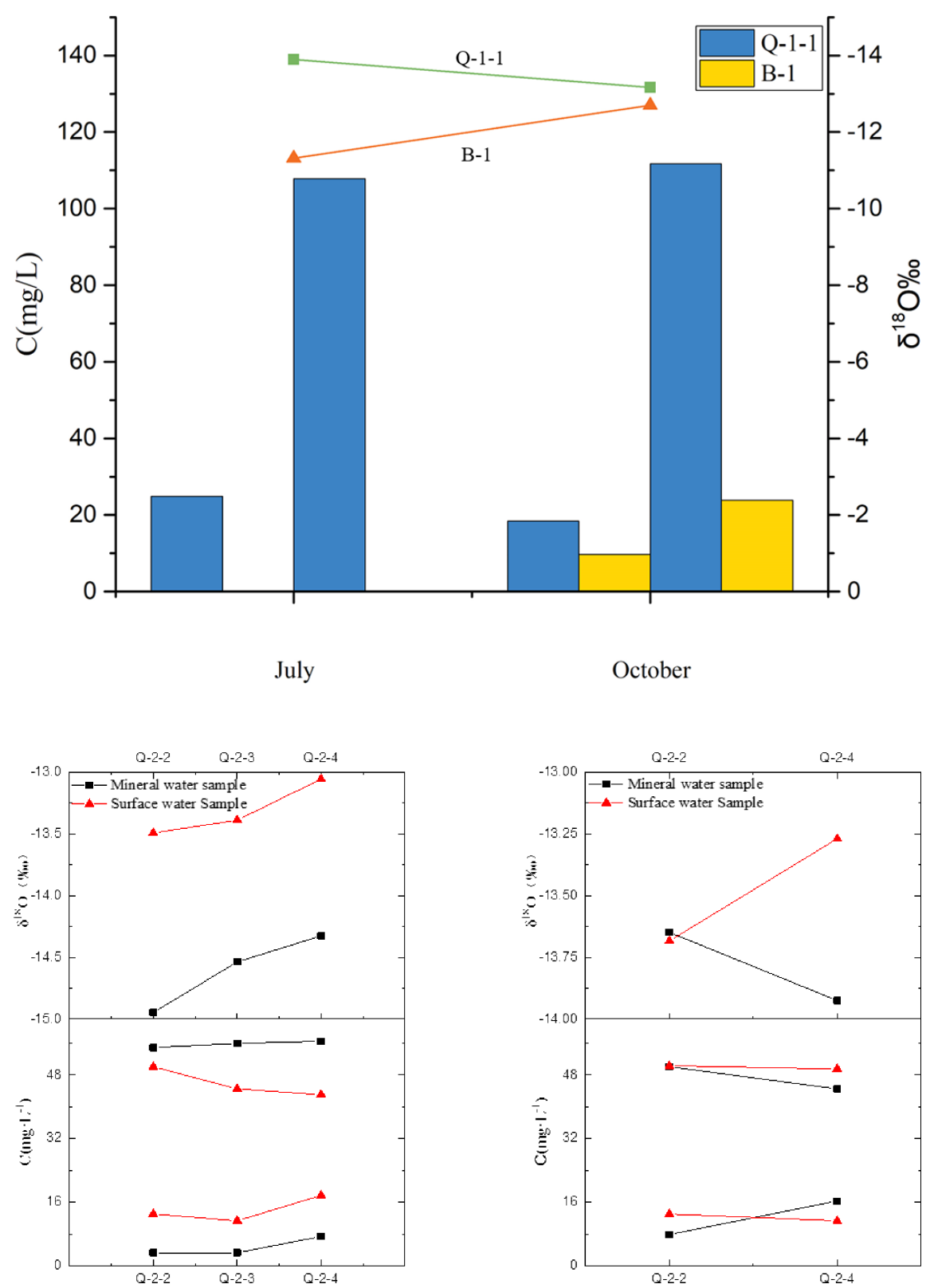

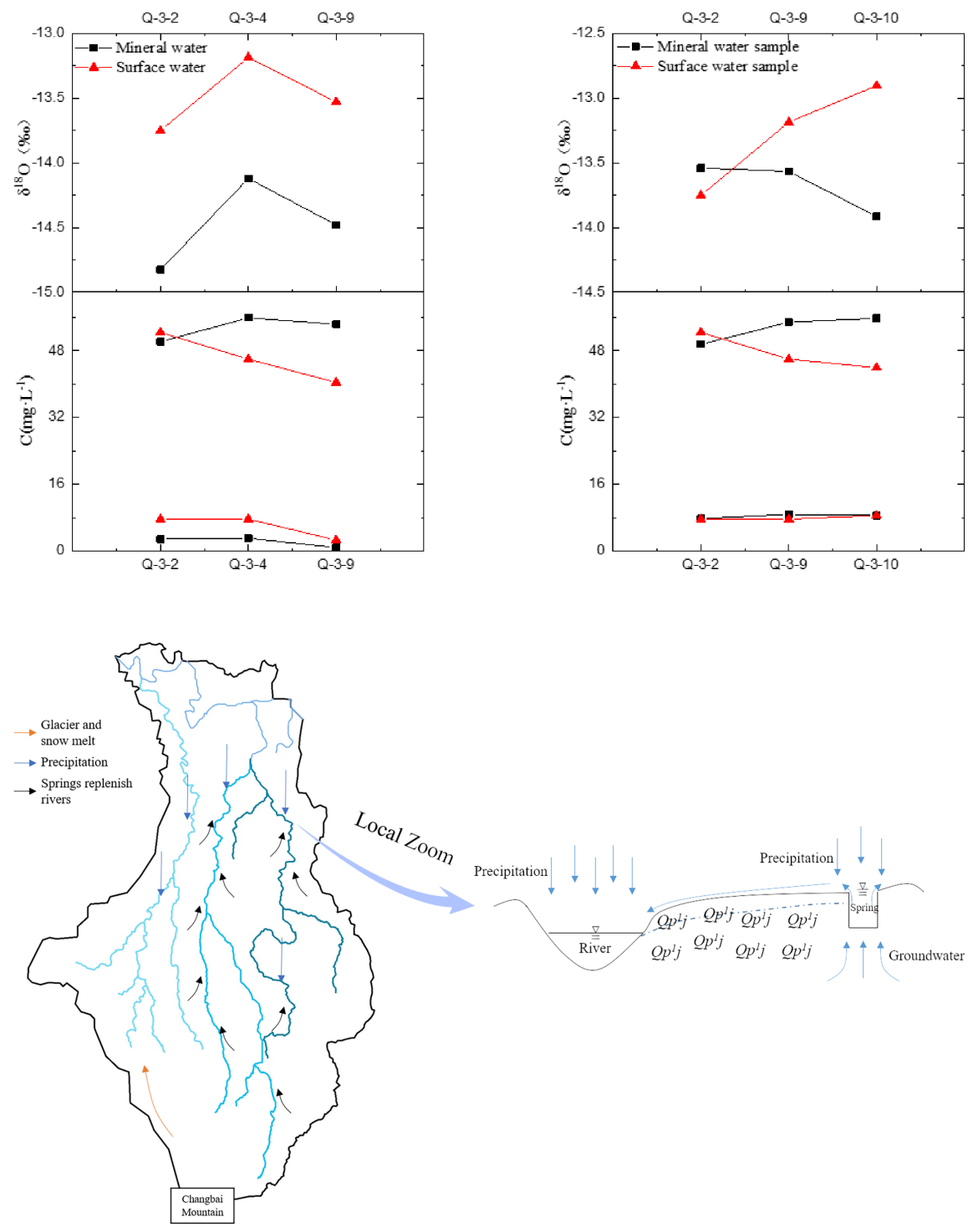

\begin{tabular}{ccccccccccccc}
\hline \multirow{2}{*}{ Year } & $\begin{array}{c}\text { Statistical } \\
\text { Index }\end{array}$ & \multirow{2}{*}{$\mathrm{pH}$} & \multicolumn{10}{c}{ Main Components (mg/L) } \\
\cline { 4 - 13 } & & $\mathrm{K}^{+}$ & $\mathrm{Na}^{+}$ & $\mathrm{Ca}^{2+}$ & $\mathrm{Mg}^{2+}$ & $\mathrm{HCO}_{3}{ }^{-}$ & $\mathrm{Cl}^{-}$ & $\mathrm{SO}_{4}{ }^{2-}$ & $\mathrm{TDS}$ & $\mathrm{H}_{2} \mathrm{SiO}_{3}$ & $\mathrm{~F}^{-}$ \\
\hline $2016^{*}$ & & 7.24 & 2.31 & 9.54 & 6.18 & 5.00 & 61.50 & 1.00 & 3.16 & 133.00 & 53.40 & 0.72 \\
\hline \multirow{2}{*}{2017} & Max & 7.83 & 3.18 & 8.42 & 12.60 & 6.15 & 83.30 & 1.03 & 3.66 & 166.20 & 57.50 & 0.78 \\
& Min & 7.00 & 2.52 & 7.89 & 5.40 & 3.93 & 56.10 & 0.92 & 3.29 & 129.00 & 55.80 & 0.76 \\
& Mean & 7.53 & 2.76 & 8.17 & 7.46 & 5.21 & 66.48 & 0.99 & 3.48 & 140.80 & 56.68 & 0.77 \\
\hline \multirow{2}{*}{2018} & Max & 7.89 & 3.02 & 10.81 & 16.51 & 5.60 & 74.99 & 1.27 & 7.02 & 188.00 & 59.13 & 1.34 \\
& Min & 6.96 & 2.66 & 8.26 & 4.25 & 3.33 & 51.92 & 0.90 & 2.90 & 93.80 & 52.90 & 0.68 \\
& Mean & 7.34 & 2.78 & 9.10 & 8.48 & 4.41 & 61.01 & 0.99 & 3.99 & 143.55 & 55.90 & 0.84 \\
\hline \multirow{2}{*}{2019} & Max & 7.80 & 3.07 & 11.70 & 7.49 & 4.96 & 67.80 & 1.50 & 7.10 & 130.00 & 58.10 & 1.77 \\
& Min & 7.01 & 2.75 & 7.94 & 4.07 & 3.42 & 42.00 & 0.90 & 2.73 & 95.00 & 49.60 & 0.80 \\
& Mean & 7.27 & 2.94 & 8.84 & 4.98 & 4.32 & 54.17 & 1.14 & 3.62 & 115.63 & 54.55 & 0.97 \\
\hline
\end{tabular}

*There is only one group of data collected in 2016 


\begin{tabular}{|c|c|c|c|c|c|c|c|c|c|c|c|c|}
\hline \multirow{2}{*}{$\begin{array}{l}\text { Sample } \\
\text { Type }\end{array}$} & \multirow{2}{*}{$\begin{array}{l}\text { Statistical } \\
\text { Index }\end{array}$} & \multirow{2}{*}{$\mathrm{pH}$} & \multicolumn{10}{|c|}{ Main Components (mg/L) } \\
\hline & & & $\mathrm{K}^{+}$ & $\mathrm{Na}^{+}$ & $\mathrm{Ca}^{2+}$ & $\mathrm{Mg}^{2+}$ & $\mathrm{HCO}_{3}^{-}$ & $\mathrm{Cl}^{-}$ & $\mathrm{SO}_{4}{ }^{2-}$ & TDS & $\mathrm{H}_{2} \mathrm{SiO}_{3}$ & $\mathrm{~F}^{-}$ \\
\hline \multirow{3}{*}{$\begin{array}{c}\text { Surface } \\
\text { Water Sample }\end{array}$} & Max & 7.20 & 2.62 & 6.09 & 3.51 & 1.38 & 43.92 & 2.63 & 3.27 & 98.00 & 23.89 & 0.47 \\
\hline & Min & 7.76 & 4.25 & 33.18 & 11.51 & 4.27 & 120.63 & 17.75 & 6.17 & 182.00 & 52.38 & 1.56 \\
\hline & Mean & 7.41 & 3.23 & 17.08 & 7.38 & 2.98 & 71.52 & 9.79 & 4.86 & 123.57 & 43.01 & 1.10 \\
\hline \multirow{3}{*}{$\begin{array}{c}\text { Mineral } \\
\text { Water Sample }\end{array}$} & Max & 7.54 & 2.70 & 5.83 & 3.73 & 2.41 & 32.79 & 0.84 & 1.11 & 56.00 & 23.89 & 0.25 \\
\hline & Min & 6.16 & 11.54 & 136.01 & 181.61 & 143.78 & 1317.60 & 24.85 & 7.06 & 1402.40 & 111.74 & 1.56 \\
\hline & Mean & 7.02 & 4.46 & 22.99 & 29.50 & 22.60 & 256.79 & 6.70 & 3.48 & 296.74 & 57.68 & 0.74 \\
\hline
\end{tabular}

